\title{
Lowering effects of aspirin eugenol ester on blood lipids in rats with high fat diet
}

\author{
Isam Karam ${ }^{\dagger}$, Ning Ma ${ }^{\dagger}$, Xi-Wang Liu, Xiao-Jun Kong, Xiao-Le Zhao, Ya-Jun Yang ${ }^{*}$ and Jian-Yong Li
}

\begin{abstract}
Background: Aspirin and eugenol were esterified to synthesize aspirin eugenol ester (AEE). As a pale yellow and odourless crystal, AEE reduced the gastrointestinal damage of aspirin and vulnerability of eugenol. The study was conducted to evaluate the preventive effects of AEE on blood lipids in rats with high fat diet (HFD).

Methods: Suspensions of AEE and simvastatin were prepared in 5\% carboxymethyl cellulose sodium (CMC-Na). In order to observe the intervention effects, the drugs and HFD were administrated at the same time. Based on individual weekly body weight (BW), AEE was intragastrically administrated at the dosage of 18, 36 and $54 \mathrm{mg} / \mathrm{kg}$. Simvastatin $(10 \mathrm{mg} / \mathrm{kg})$ and CMC-Na $(20 \mathrm{mg} / \mathrm{kg})$ were used as control drug. After 6 weeks of administration, the changes of BW and blood lipid indices including triglyceride (TG), low density lipoprotein (LDL), high density lipoprotein (HDL) and total cholesterol (TCH) were determined in the experiment.

Results: The rat blood lipids profile in model group was remarkably different after feeding 6-weeks HFD. TG, TCH and LDL indexes in model group were increased significantly compared with those in control group $(p<0.01)$. AEE at the dosage of $54 \mathrm{mg} / \mathrm{kg}$ significantly decreased levels of TG, TCH and LDL $(p<0.01)$, and slowed the rate of BW gain in comparison with model group $(p<0.05)$. Moreover, high dose AEE showed better effects than simvastatin on reducing TCH level and similar effects on TG, HDL and LDL.

Conclusion: AEE could remarkably reduce levels of TG, TCH and LDL in rats with high fat diet, and slow the rate of body weight gain. It was conducted that AEE was a potential candidate on reducing blood lipids level. The mechanism of action of AEE should be investigated in further studies.
\end{abstract}

Keywords: Aspirin eugenol ester (AEE), Blood lipids, Body weight, High fat diet, Rats

\section{Background}

Hyperlipidemia is a heterogeneous group of disorders characterized by an excess of lipids in blood stream such as the increased serum levels of triglycerides (TG), total cholesterol (TCH), low-density lipoprotein (LDL) as well as decreased levels of high-density lipoprotein (HDL) $[1,2]$. Hyperlipidemia, as the major risk factor for the development of cardiovascular diseases, is becoming a major health problem in the world [3].

Aspirin could ameliorate hyperlipidemia induced by high fat diet and hyperinsulinemia in rats [4]. Moreover, aspirin could diminish hypertriglyceridemia in obese

\footnotetext{
*Correspondence: yangyue10224@163.com; lijy1971@163.com 'Equal contributors

Key Lab of New Animal Drug Project of Gansu Province, Key Lab of Veterinary Pharmaceutical Development, Ministry of Agriculture, Lanzhou Institute of Husbandry and Pharmaceutical Science of CAAS, No.335, jiangouyan, qilihe district, Lanzhou 730050, China
}

rodents and has potential in hyperlipidemia prevention [5]. Eugenol as volatile oil is extracted from dry alabastrum of Eugenia caryophyllata. Therapeutic effects of eugenol on hyperlipidemia had been proved in previous study [6, 7]. Four-week administration of eugenol could significantly decrease the serum lipid profile in normal albino rabbits [8].

Based on prodrug principle and therapeutic effects of aspirin and eugenol on hyperlipidemia, aspirin eugenol ester (AEE) as a new drug was synthesized [9]. The metabolites of AEE had been confirmed in beagle dog and liver microsomes. AEE could be metabolized into aspirin and eugenol in vitro and in vivo, which could show their original activities and act synergistically [10]. AEE also reduced the side effects of its precursor such as the gastrointestinal damage of aspirin and irritation of eugenol [11]. The acute toxicity of AEE was less than its 
precursor, which was 0.02 times of aspirin and 0.27 times of eugenol $[9,11]$. The teratogenicity and mutagenicity of AEE have been investigated. AEE did not show any mutagenesis in Ames test and the mouse bone marrow micronucleus assay $[11,12]$. Moreover, the effects of AEE had been evaluated in animal model. The results showed that AEE had positive effects on antithrombosis, anti-inflammatory, analgesia and antipyretic $[9,11,13]$.

Therapeutic strategies for hyperlipidemia treatment depend on reducing blood lipids. There are many chemical drugs that lower cholesterol level in the body such as statins, fibrates, ezetimibe and nicotinic acid. However, most of them are expensive and have undesirable effects [14]. There is an obvious need for more efficacious and alternative treatment options for hyperlipidemia. In our previous study, AEE $(50 \mathrm{mg} / \mathrm{kg}$ and $160 \mathrm{mg} / \mathrm{kg}$ ) could reduce $\mathrm{TCH}$ and $\mathrm{TG}$ in rats with standard diet [11]. Moreover, five-week administration of AEE (54 mg/kg) could normalize blood lipids profile in hyperlipidemic rats [15]. So there are increasing interest to evaluate the intervention effects of AEE on blood lipids in rat with high fat diet. This study will increase the understanding of $\mathrm{AEE}$ and provide impetus for further studies.

\section{Methods}

\section{Chemicals and reagents}

Aspirin eugenol ester (AEE), transparent crystal with the purity of $99.5 \%$ by RE-HPLC, was prepared in Key Lab of New Animal Drug Project of Gansu Province, Key Lab of Veterinary Pharmaceutical Development of Agricultural Ministry, Lanzhou Institute of Husbandry and Pharmaceutical Sciences of CAAS. CMC-Na and simvastatin was supplied by Tianjin Chemical Reagent Company (Tianjin, China). Standard compressed rat feed and high diet food were supplied by Keao Xieli Co., Ltd (Beijing, China). Standard rat diet consisted of $12.3 \%$ lipids, $63.3 \%$ carbohydrates, and $24.4 \%$ proteins (kcal) and high fat diet (standard rat diet $77.8 \%$, yolk power $10 \%$, lard $10 \%$, cholesterol $2 \%$, bile salts $0.2 \%$ ) consisted of $41.5 \%$ lipids, $40.2 \%$ carbohydrates, and $18.3 \%$ proteins (kcal). The TG, TCH, LDL and HDL kits were provided by Ningbo Medical System Biotechnology Co., Ltd (Ningbo, China). Erba XL-640 analyzer (German) was used to measure the blood lipid indices.

\section{Animals}

Seventy Sprague-Dawley (SD) male rats were purchased from the animal breeding facilities of Gansu University of Chinese Medicine (Lanzhou, China). The rats were housed in plastic cages of appropriate size $(50 \times 35 \times$ $20 \mathrm{~cm}$, ten rats per cage) with stainless steel wire cover and chopped bedding. Rat feed and drinking water were supplied ad libitum. Light/dark regime was 12/12 h and living temperature was $22 \pm 2{ }^{\circ} \mathrm{C}$ with relative humidity of $55 \pm 10 \%$. Animals were acclimatized for 2 weeks before study initiation.

\section{Serum sampling}

At the end of the experiment, rats were fasted for 10-12 $\mathrm{h}$ and then anaesthetized with $1 \%$ pentobarbital sodium. The blood samples were collected from the heart with vacuum tube. The sera were obtained by centrifuging for 15 mins at the speed of $4000 \mathrm{~g}$ at $4{ }^{\circ} \mathrm{C}$. Serum samples were stored at $-80{ }^{\circ} \mathrm{C}$ until the day of analysis.

\section{Drug preparation}

$\mathrm{AEE}$ and simvastatin liquid suspensions were prepared in $0.5 \%$ of $\mathrm{CMC}-\mathrm{Na}$.

\section{Study design}

Rats were randomly divided into seven groups including control, model, CMC-Na, simvastatin and three AEE groups. The detailed design of the experiment is shown in Table 1. Simvastatin was used as a positive control $(10 \mathrm{mg} / \mathrm{kg})$. The administrations of food and drugs were started at the same time. The volumes of $\mathrm{CMC}-\mathrm{Na}$ and drug suspensions were nearly equal. After 6-weeks administration, the blood lipid levels were analyzed.

\section{Statistics}

The statistical analyses were carried out using IBM SPSS 19.0 (USA). All data obtained from the experiment are expressed as mean \pm standard deviation (SD). Statistical differences were evaluated by using one-way ANOVA with Tukey's multiple comparison tests. P-values less than 0.05 were considered statistically significant.

Table 1 Study design of the experiment

\begin{tabular}{lllll}
\hline Groups & Food & Drug & Dosage & Concentration \\
\hline Control & SRD & - & - & - \\
Model & HFD & - & - & - \\
CMC-Na & HFD & CMC-Na & $20 \mathrm{mg} / \mathrm{kg}$ & $5 \mathrm{mg} / \mathrm{mL}$ \\
Statin & HFD & Simvastatin & $10 \mathrm{mg} / \mathrm{kg}$ & $2.5 \mathrm{mg} / \mathrm{mL}$ \\
AEE Low & HFD & AEE & $18 \mathrm{mg} / \mathrm{kg}$ & $4.5 \mathrm{mg} / \mathrm{mL}$ \\
AEE Mid & HFD & AEE & $36 \mathrm{mg} / \mathrm{kg}$ & $9 \mathrm{mg} / \mathrm{mL}$ \\
AEE High & HFD & AEE & $54 \mathrm{mg} / \mathrm{kg}$ & $13.5 \mathrm{mg} / \mathrm{mL}$
\end{tabular}

Rats were divided into seven groups. Control group was received SRD and the rest groups were received HFD, respectively. Based on the individual weekly body weight, rats were given with different drug suspension volume. CMC-Na as a vehicle was used in control group. Simvastatin was designed as positive drug. Different dose of three levels of AEE were administrated in the study. The administration period was 6 weeks and then blood lipids were analyzed $A E E$ aspirin eugenol ester, SRD standard rat diet, HFD high fat diet 


\section{Results}

\section{Intervention effects of AEE}

After feeding rats with HFD for 6 weeks, the blood lipid profile was notably different among the groups. There were significant differences between control and model groups (Table 2). In model group, TG, TCH and LDL were increased significantly in comparison with the control group $(p<0.01)$. However, no change was observed in HDL between control and model groups. There was no significant difference between CMC-Na and model groups. In comparing with model group, low and intermediate dose of AEE had no effect on blood lipid indexes except $\mathrm{TCH}$ index in AEE low group $(p<0.05)$. AEE high dose significantly decreased TG, TCH and LDL compared to model group $(p<0.01)$. AEE in different dosages had no influence on HDL. Simvastatin as a positive drug control could significantly decrease $\mathrm{TCH}$ and LDL $(p<0.01)$. With regard to TG and HDL, no changes were observed between simvastatin and model groups.

The three groups were used to compare with control and statin groups (Fig. 1). When compared with control group, TG, TCH and LDL in AEE groups were significant increased $(p<0.01$ or $p<0.05)$. Notably, the mean values of TG, TCH and LDL in AEE high group were less than the values in low and intermediate AEE groups. Simvastatin and AEE made similar effects on TG, HDL and LDL indexes. However, simvastatin showed more positive effects on TCH than AEE L and AEE M but not AEE $\mathrm{H}(p<0.01$ or $p<0.05$ Fig. $1 \mathrm{~d})$. In order to find out the relationship between drug effects and the dosage, intermediate and high AEE groups were compared with low dose AEE group. The results showed that there was only significant difference on $\mathrm{TCH}$ index in AEE high group ( $p<0.01$ Fig. $1 \mathrm{~d}$ ). Interestingly, the mean values of TG and LDL in AEE high group were the smallest in all AEE groups.

\section{Body weight}

The body weights of all groups were recorded at the beginning and ending of the experiment (Table 3). At the beginning of the experiment, there was no significant difference between all groups. At week 6, the body weights in the group supplemented with AEE high dose were significantly lower than those in model group $(p<$ $0.05)$. The results showed that high dose of AEE could reduce the rate of body weight gain. The body weights in AEE intermediate and high groups were remarkably lower than control group $(p<0.05$ or $p<0.01)$. No difference was observed among statin and AEE groups. The gains in body weights in AEE high group were significantly lower than those in AEE low group $(p<0.05)$.

\section{Discussion}

Based on prodrug principle, aspirin and eugenol, as starting precursors, were esterified to synthesize aspirin eugenol ester (AEE). The positive effects of AEE on the symptoms of inflammation, fever, pain and thrombosis had been confirmed in animal disease model $[9,11,13]$. The blood chemistry results in 15-day oral dose toxicity study showed AEE could significantly reduce the values of TG and TCH both in male and female rat, which indicated that this compound was potential for curing hyperlipidemia [11]. In subsequent experiment, the regulation effects of AEE on blood lipid profile in hyperlipidemic rats had been confirmed [15]. Based on this fact, it is essential to evaluate the intervention effects of AEE on blood lipids in rats with high fat diet fed.

The intervention effect of AEE was evaluated by comparing AEE groups with model group. AEE low and intermediate dose showed no significant effect on blood lipid indices except reducing TCH at AEE low dose. It is noteworthy that high dose of AEE was highly effective in reducing blood lipid indices such as TG and $\mathrm{TCH}$. These meant that the activity of AEE at high dose was better than low and intermediate doses. So there was a positive relationship between drug efficacy and dosage. Under the present experimental conditions, the optimal dose was considered to be $54 \mathrm{mg} / \mathrm{kg}$ for normalizing blood lipid profile in high fat diet fed rat.

HDL is responsible for the transportation of cholesterol to liver, which is essential for cholesterol removal [16]. HDL levels of rat fed with high fat diet for six weeks showed no changes in this study. There are several possible reasons for this result. First of all, HFD compositions such as the lack of saturated fat may be insufficient to make substantial change on HDL. Second, the constant values of HDL may be attributable to the

Table 2 The blood lipids levels after drugs administration for six weeks $(n=10)$

\begin{tabular}{|c|c|c|c|c|c|c|c|}
\hline Variables & Control & Model & CMC-Na & Statin & AEE LOW & AEE Mid & AEE High \\
\hline$\overline{\mathrm{TG}}(\mathrm{mmol} / \mathrm{L})$ & $0.53 \pm 0.12^{* *}$ & $0.89 \pm 0.07$ & $0.79 \pm 0.19$ & $0.83 \pm 0.05$ & $0.79 \pm 0.23$ & $0.77 \pm 0.10$ & $0.71 \pm 0.07^{* *}$ \\
\hline $\mathrm{HDL}(\mathrm{mmol} / \mathrm{L})$ & $0.72 \pm 0.04$ & $0.74 \pm 0.08$ & $0.69 \pm 0.09$ & $0.66 \pm 0.13$ & $0.73 \pm 0.09$ & $0.79 \pm 0.10$ & $0.65 \pm 0.15$ \\
\hline LDL (mmol/L) & $0.25 \pm 0.02^{* *}$ & $0.50 \pm 0.09$ & $0.46 \pm 0.06$ & $0.39 \pm 0.13^{* *}$ & $0.45 \pm 0.05$ & $0.46 \pm 0.10$ & $0.36 \pm 0.08^{* *}$ \\
\hline $\mathrm{TCH}(\mathrm{mmol} / \mathrm{L})$ & $1.4 \pm 0.10^{* *}$ & $2.29 \pm 0.26$ & $2.13 \pm 0.22$ & $1.80 \pm 0.16^{* *}$ & $2.10 \pm 0.22^{*}$ & $2.15 \pm 0.15$ & $1.66 \pm 0.15^{* *}$ \\
\hline
\end{tabular}

${ }^{*} P<0.05,{ }^{* *} P<0.01$ significant difference compared to model group. $T G$ triglyceride, $H D L$ high density lipoprotein, $L D L$ low density lipoprotein, $T C H$ total cholesterol. Blood lipids indices were increased in model group. The blood lipid levels were reduced in simvastatin and high dose AEE groups in varying degrees 
a

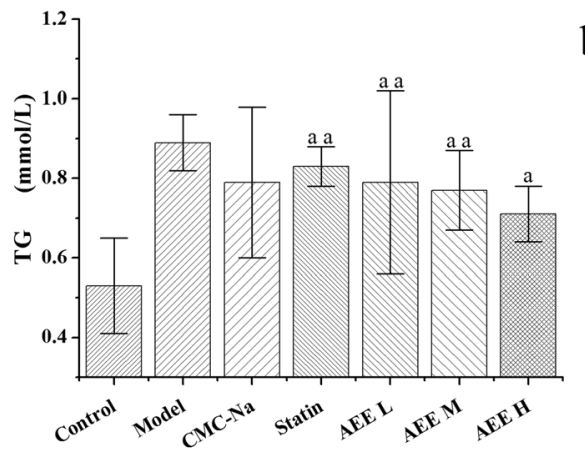

c

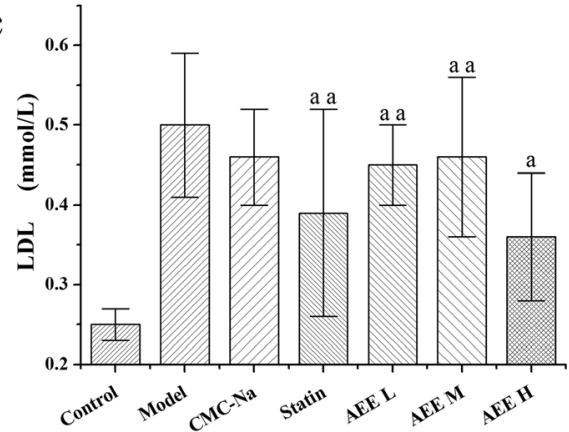

b

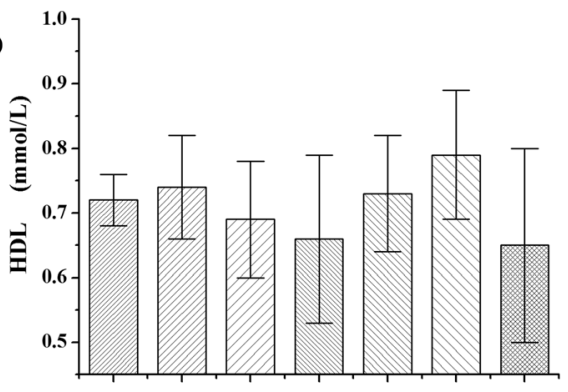

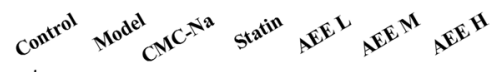

d

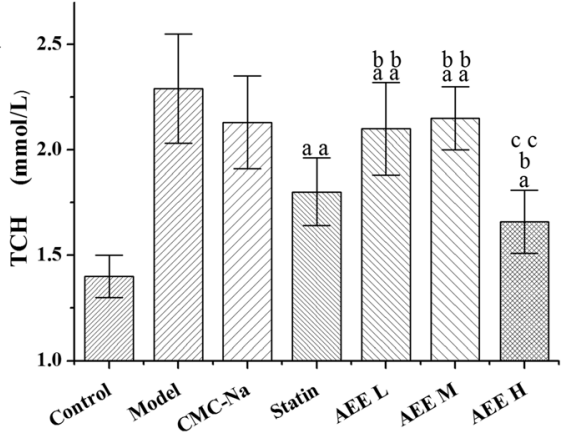

Fig. 1 Comparative effects of AEE and simvastatin on blood lipid indexes $(n=10)$. ${ }^{a} p<0.05$, aa $p<0.01$ significant difference compared to control group; ${ }^{b} P<0.05,{ }^{b b} P<0.01$ significant difference compared to statin group; ${ }^{c} P<0.05,{ }^{c c} P<0.01$ significant difference compared to AEE L. CMC-Na: carboxymethylcellulose sodium, AEE L: low dose of AEE (18 mg/kg), AEE M: intermediate dose of AEE (36 mg/kg), AEE H: high dose of AEE $(54 \mathrm{mg} / \mathrm{kg})$. a, b, c and $\mathbf{d}$ were $\mathrm{TG}, \mathrm{HDL}, \mathrm{LDL}$ and $\mathrm{TCH}$, respectively

short duration of the experiment (only 6 weeks). Finally, animal model used in the experiment may be a potential cause. Literature indicated that most cholesterol in rat blood circulation existed in HDL and this contributed to reducing susceptibility of HDL level to $\operatorname{HFD}[17,18]$.

HFD is a commonly used material to induce animal disease such as hyperlipidemia, diabetes, obesity and arteriosclerosis $[19,20]$. In this experiment, the results of body weight were not consistent with those in our previous study. There was no difference in body weight between control and model group. However, HFD with same components significantly increased body weight at

Table 3 Body weights of rats at the beginning and the end of the experiment $(n=10)$

\begin{tabular}{lll}
\hline Groups & Week 1 & Week 6 \\
\hline Control & $209 \pm 15$ & $385 \pm 20$ \\
Model & $216 \pm 11$ & $368 \pm 25$ \\
CMC-Na & $209 \pm 10$ & $365 \pm 20$ \\
Statin & $220 \pm 22$ & $355 \pm 20^{\mathrm{a}}$ \\
AEE Low & $220 \pm 17$ & $366 \pm 31$ \\
AEE Mid & $211 \pm 15$ & $356 \pm 20^{\mathrm{a}}$ \\
AEE High & $213 \pm 14$ & $340 \pm 15^{* \text { aab }}$
\end{tabular}

${ }^{\overline{ }} P<0.05$ significant difference compared to model group; ${ }^{\mathrm{a}} P<0.05$, ${ }^{\text {aa }} P<0.01$ significant difference compared to control group; ${ }^{\mathrm{b}} P<0.05$ significant difference compared to AEE low group; Week 1: beginning of the experiment; Week 6: the end of the experiment the end of eighth week in Wistar rat [15]. The shortage of HFD consumption time may be the reason for the results of no significant difference in body weight. Metabolism and liver function could be substantially changed with HFD feeding [21]. It was necessary to give the rat time to adjust for HFD gradually, which may slow the rate of the body weight gain in certain period. It was found that AEE dosage had different influence on body weight. AEE high and middle dose made an impact on body weight and there was significant difference between low and high AEE groups. The results of AEE on body weight were also the reason why $54 \mathrm{mg} / \mathrm{kg}$ was suggested for optimal dosage.

Simvastatin is an inhibiter of 3-hydroxy-3-methylglutaryl coenzyme A (HMG-CoA) which plays a crucial role in cholesterol synthesis in liver cells. Simvastatin was used as a positive drug control in this study and it decreased the levels of TCH and LDL. The effects of $10 \mathrm{mg} / \mathrm{kg}$ simvastatin on $\mathrm{TCH}$ and LDL were similar with $54 \mathrm{mg} / \mathrm{kg}$ AEE. However, simvastatin had no influence in TG index and showed significant difference from high dose AEE. From these results, it could be concluded that AEE was more effective than simvastatin on reducing blood lipid level. CMC-Na was widely used as a reliable drug vehicle in pharmaceutical industry [22, 23]. The effect of CMC-Na on blood lipid indexes was eliminated by administrating equal volume $\mathrm{CMC}-\mathrm{Na}$ to 
control group. Therefore, it manifested that the effects of AEE was not related to CMC-Na.

Therefore, intragastrical administration of AEE had a significant intervention effects in HFD fed rat. AEE was decomposed into salicylic acid and eugenol after absorption. The effects of AEE on reducing blood lipid indexes may be mainly from synergetic action of aspirin and eugenol. More studies are necessary to investigate the action mechanism of AEE such as evaluation of inhibitory effect on digestive enzymes and influence on metabolic targets.

\section{Conclusions}

Under the present study condition, AEE at daily dosage of $54 \mathrm{mg} / \mathrm{kg} \mathrm{BW}$ for six weeks could remarkably reduce levels of TG, TCH and LDL in rats with high fat diet, and slow the rate of body weight gain. Moreover, AEE at $54 \mathrm{mg} / \mathrm{kg}$ showed better effects than simvastatin at $10 \mathrm{mg} / \mathrm{kg}$ on reducing $\mathrm{TCH}$ level and similar effects on TG, HDL and LDL.

\section{Abbreviations}

AEE: Aspirin eugenol ester; BW: Body weight; CMC-Na: Sodium carboxymethyl cellulose; HDL: High density lipoprotein; HFD: High fat diet; LDL: Low density lipoprotein; SRD: Standard rat diet; TCH: Total cholesterol; TG: Triglycerides

\section{Acknowledgements}

The work was supported by the National Natural Science Foundation of China (No.31402254) and the Natural Science Foundation for Youth of Gansu Province (1506RJYA148).

\section{Funding}

The funders had no role in the design of the study and collection, analysis, and interpretation of data and in writing the manuscript.

\section{Availability of data and materials}

All data generated or analyzed during the current study are available from the corresponding author on reasonable request.

\section{Authors' contributions}

Conceived and designed the experiments: NM JYL. Performed the experiments: NM IM ZXL. Analyzed the data: NM YJY XWL IM. Contributed reagents/materials/analysis tools: IM NM XWL XJK. Wrote the paper: NM YJY $J Y L$. All authors read and approved the final manuscript.

\section{Competing interests}

The authors have declared that no competing interests existed.

\section{Consent for publication}

Not applicable.

\section{Ethics approval}

The study protocol was approved by the Institutional Animal Care and Use Committee of Lanzhou Institute of Husbandry and Pharmaceutical Science of the Chinese Academy of Agricultural Sciences. Animal welfare and experimental procedures were performed strictly in accordance with the Guidelines for the Care and Use of Laboratory Animals issued by the US National Institutes of Health.

Received: 4 September 2016 Accepted: 10 November 2016 Published online: 17 November 2016

\section{References}

1. Xu C, Wang X, Wang S. Effect of soybean protein and high calcium intake on the concentration of serum lipids in hypercholesterolemic rats. Zhonghua Yu Fang Yi Xue Za Zhi. 2001;35(5):318-21.

2. Graham JM, Higgins JA, Taylor T, Gillott T, Wilkinson J, Ford TC, et al. A novel method for the rapid separation of human plasma lipoproteins using self-generating gradients of lodixanol. Biochem Soc Trans. 1996;24(2):170S.

3. Forouzanfar MH, Alexander L, Anderson HR, Bachman VF, Biryukov S, Brauer $\mathrm{M}$, et al. Global, regional, and national comparative risk assessment of 79 behavioural, environmental and occupational, and metabolic risks or clusters of risks in 188 countries, 1990-2013: a systematic analysis for the Global Burden of Disease Study 2013. Lancet. 2015;386(10010):2287-323.

4. Lin HL, Yen HW, Hsieh SL, An LM, Shen KP. Low-dose aspirin ameliorated hyperlipidemia, adhesion molecule, and chemokine production induced by high-fat diet in Sprague-Dawley rats. Drug Dev Res. 2014;75(2):97-106.

5. Yuan M, Konstantopoulos N, Lee J, Hansen L, Li ZW, Karin M, et al. Reversal of obesity- and diet-induced insulin resistance with salicylates or targeted disruption of Ikkbeta. Science. 2001;293(5535):1673-7.

6. Suanarunsawat T, Boonnak T, Na AW, Thirawarapan S. Anti-hyperlipidemic and cardioprotective effects of Ocimum sanctum L. fixed oil in rats fed a high fat diet. J Basic Clin Physiol Pharmacol. 2010;21(4):387-400.

7. Suanarunsawat T, Ayutthaya WD, Songsak T, Thirawarapan S, Poungshompoo S. Lipid-lowering and antioxidative activities of aqueous extracts of Ocimum sanctum L. leaves in rats fed with a high-cholesterol diet. Oxid Med Cell Longev. 2011;2011:962025.

8. Sarkar A, Lavania SC, Pandey DN, Pant MC. Changes in the blood lipid profile after administration of Ocimum sanctum (Tulsi) leaves in the normal albino rabbits. Indian J Physiol Pharmacol. 1994;38(4):311-2.

9. Li J, Yu Y, Wang Q, Zhang J, Yang Y, Li B, et al. Synthesis of aspirin eugenol ester and its biological activity. Med Chem Res. 2012;21(7):995-9.

10. Shen Y, Liu X, Yang Y, Li J, Ma N, Li B. In vivo and in vitro metabolism of aspirin eugenol ester in dog by liquid chromatography tandem mass spectrometry. Biomed Chromatogr. 2015;29(1):129-37.

11. Li J, Yu Y, Yang Y, Liu X, Zhang J, Li B, et al. A 15-day oral dose toxicity study of aspirin eugenol ester in Wistar rats. Food Chem Toxicol. 2012;50(6):1980-5.

12. Li J, Kong X, Li X, Yang Y, Zhang J. Genotoxic evaluation of aspirin eugenol ester using the Ames test and the mouse bone marrow micronucleus assay. Food Chem Toxicol. 2013;62:805-9.

13. Ma N, Liu XW, Yang YJ, Li JY, Mohamed I, Liu GR, et al. Preventive Effect of Aspirin Eugenol Ester on Thrombosis in kappa-Carrageenan-Induced Rat Tail Thrombosis Model. PLoS One. 2015;10(7):e133125.

14. Thomas HD, Maynard C, Wagner GS, Eisenstein EL. Results from a practicebased lipid clinic model in achieving low density lipoprotein cholesterol goals. N C Med J. 2003;64(6):263-6.

15. Karam I, Ma N, Liu XW, Li SH, Kong XJ, Li JY, et al. Regulation effect of Aspirin Eugenol Ester on blood lipids in Wistar rats with hyperlipidemia. BMC Vet Res. 2015;11:217.

16. Wang Z, Li P, Wang C, Jiang Q, Zhang L, Cao Y, et al. Protective effects of Arctium lappa L. root extracts (AREs) on high fat diet induced quail atherosclerosis. BMC Complement Altern Med. 2016;16(1):6.

17. Rosini TC, Silva AS, Moraes C. Diet-induced obesity: rodent model for the study of obesity-related disorders. Rev Assoc Med Bras. 2012;58(3):383-7.

18. Xu S, Liu Z, Liu P. HDL cholesterol in cardiovascular diseases: The good, the bad, and the ugly? Int J Cardiol. 2013;168(4):3157-9.

19. Bakillah A, Hussain MM. Mice subjected to aP2-Cre mediated ablation of microsomal triglyceride transfer protein are resistant to high fat diet induced obesity. Nutr Metab (Lond). 2016;13:1

20. Jugdaohsingh R, Kessler K, Messner B, Stoiber M, Pedro LD, Schima H, et al. Dietary Silicon Deficiency Does Not Exacerbate Diet-Induced Fatty Lesions in Female ApoE Knockout Mice. J Nutr. 2015;145(7):1498-506.

21. Hao L, Lu X, Sun M, Li K, Shen L, Wu T. Protective effects of L-arabinose in high-carbohydrate, high-fat diet-induced metabolic syndrome in rats. Food Nutr Res. 2015:59:28886.

22. Jin Z, Li W, Cao H, Zhang X, Chen G, Wu H, et al. Antimicrobial activity and cytotoxicity of N-2-HACC and characterization of nanoparticles with $\mathrm{N}-2$ HACC and CMC as a vaccine carrier. Chem Eng J. 2013;221:331-41.

23. Abdel-Galil A, Ali HE, Atta A, Balboul MR. Influence of nanostructured TiO2 additives on some physical characteristics of carboxymethyl cellulose (CMC). J Radiat ResAppl Sci. 2014;7(1):36-43. 